\title{
Mitchell Feigenbaum
}

\section{Konstantin Khanin, Mikhail Lyubich, Eric D. Siggia, and Yakov Sinai}

\section{Preamble}

Mitchell Feigenbaum passed away on June 30, 2019. His profound discoveries opened a new page in mathematics and physics, in their fascinating intertwining. Volumes can be already written on this subject. In the notes below we give a brief retrospective of the impact of Feigenbaum's ideas on (mostly) mathematics, flavored with some personal recollections.

\section{Mitchell Feigenbaum: Naturphilosoph and a Friend}

\section{Konstantin Khanin and Yakov Sinai}

About 45 years ago Mitchell Feigenbaum made a beautiful mathematical discovery. So beautiful and unexpected that for a while, many mathematicians refused to believe it. His discovery attracted a lot of public attention. It is quite rare when a mathematical discovery fascinates the general public. In the last 50 years, we can remember only two other stories: Wiles's proof of Fermat's Last Theorem and Perelman's solution to the Poincare conjecture. With time, the public interest heated by Mitchell's enigmatic personality cooled down. But the mathematical importance of Feigenbaum's work has only increased. In these short notes we will try to reflect on the background of Mitchell's discovery,

Konstantin Khanin is a professor of mathematics at the University of Toronto. His email address is khanin@math. toronto.edu.

Yakov Sinai is a professor of mathematics at Princeton University. His email address is sinai@math.princeton.edu.

Communicated by Notices Associate Editor Daniela De Silva.

For permission to reprint this article, please contact:

reprint-permission@ams . org.

DOI: https://doi.org/10.1090/noti2283

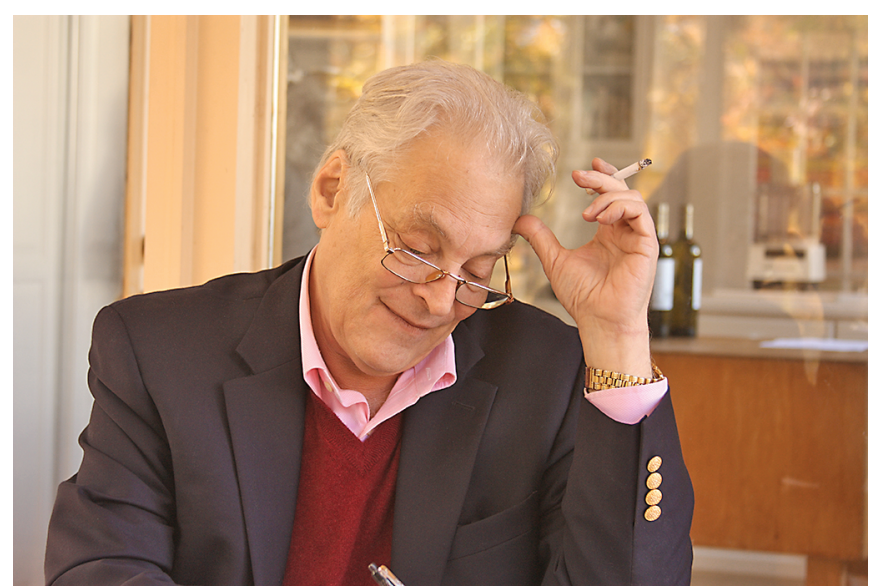

and recall the story of its early days (the late 1970s and the early 1980s). We shall also try to remember how Mitchell's many visits to the Soviet Union in the 1980s and the beginning of the 1990s resulted in our friendship which lasted almost 40 years.

Beginning. The idea of the Renormalization Group (RG) originated from condensed matter physics and statistical mechanics in the 1960s. It was a major breakthrough made in the context of the theory of phase transitions and critical phenomena. Initially developed in works by M. Fisher, L. Kadanoff, V. Pokrovsky, and A. Patashinsky centered around the ideas of scaling invariance at the point of phase transition. Later, in work by K. Wilson, it became a powerful tool in the study of critical phenomena. This later development brought forward the fundamental ideas of universality, and later in the early 1970s ideas of conformal invariance proposed by A. Polyakov. There is a huge physical literature dedicated to RG, but we are not going to go any further in this direction. However, it is important to mention that 50 years after the creation of RG theory there are very few rigorous mathematical results confirming it. It turns out that this extremely general and conceptual theory has proved elusive to mathematical approaches. 
While two beautiful theories, namely 2D conformal field theory and the theory of SLE, allowed for a lot of progress in describing the limiting objects, so-called fixed points of RG, proving convergence to such limits remains out of reach at present except for a very few concrete models. Any progress in this direction would be a great achievement. It is worth noting that one of the 2010 Fields Medals was awarded to S. Smirnov for a proof of convergence for the 2D Ising model and the critical percolation model on the planar triangular lattice.

Forgetting for a moment about mathematical rigor, one can formulate the main renormalization principle in an extremely general form. So general, that it can probably apply not only to physical and mathematical systems, but possibly also to complex systems of another nature, such as biological and social. Suppose by varying a parameter or parameters, a complex system passes through a dramatic change in its structural behavior (phase transition). Then for parameters corresponding to a point of transition, the system will exhibit certain scale invariant behavior. Moreover, very often this scaling invariant behavior has strong universality features. The above principle is formulated (intentionally) so generally and vaguely that many scientists may say that it makes no sense. We shall argue though that it has a value as an ideology, as a possible language and a way to structure our thinking about very complex phenomena.

Back to physics, by the mid 1970s the RG became a mainstream part of theoretical physics. Whenever a physicist would see a universal scaling behavior, she or he would think about the RG. The above sentence summarizes the historical physical background of the discovery made by Mitchell. The mathematical background was also very fertile. The theory of dynamical systems was very active and on the rise. In the 1960s and the beginning of the 1970s three major theories were developed and made their way into the wider mathematical community: the KAM theory dealing with elliptic dynamics (A. N. Kolmogorov, V. Arnold, J. Moser), the theory of uniformly hyperbolic systems (S. Smale, D. Anosov, Ya. Sinai, to name a few), and the thermodynamic formalism and the theory of SRB measures (Ya. Sinai, D. Ruelle, R. Bowen). It was realized that very simple deterministic systems, like the logistic family of maps $f_{a}(x)=a x(1-x)$, may exhibit very complex chaotic dynamical behavior. The ideas of chaos and dynamical chaos were becoming increasingly popular among mathematicians and physicists alike. The old idea of L. Landau to explain turbulence through a sequence of well understood dynamical bifurcations was reincarnated in the paper by D. Ruelle and F. Takens. One-dimensional dynamics was on the rise, remaining largely topological though. The rich dynamical behavior encoded by the parameter dependence in the logistic family was studied. In particular, the sequence of period-doubling bifurcations for an increasing sequence of parameter values $a_{n}$ was looked at.

This is a very brief and incomplete description of the scientific landscape in 1975 when Mitchell made his discovery. It was really a discovery, an unexpected find. A lot was said about Mitchell playing with a calculator to see for himself what the bifurcation values of parameter $a_{n}$ are for two different families of maps. He noticed that the accumulation rate looked the same. That was the moment which determined all that followed. It was luck, of course. One has to be in the right place at the right time. But one also has to be able to recognize the importance of the discovery. Not only to see how amazing it is, but also to put it into the right context. We want to add that Feigenbaum's discovery was one of the first, probably the first, meaningful and remarkable instance of what is now called experimental mathematics. It is fitting that the discovery was made by Mitchell who was working at the Theoretical Division of the Los Alamos National Lab. The group of young brilliant researchers, such as David Campbell and Predrag Cvitanović shared Feigenbaum's excitement. From the moment of discovery things moved fast. Predrag contributed by suggesting the precise neat form of the fixed point equation. Soon the paper, describing the phenomenon and providing the Renormalization Group explanation of it, was ready.

But mathematics was not yet ready to accept it. It was rejected at least twice until Joel Lebowitz published Feigenbaum's seminal paper in his Journal of Statistical Physics. Mitchell told us a story of his conversation with Jurgen Moser back then. Feigenbaum was still completely unknown in the mathematical community. He said something about period-doubling bifurcations for families of maps. Yes, it is a well-known bifurcation, Moser replied. Feigenbaum: "But I am studying sequences, in fact, infinite sequences of such bifurcations." Moser: "Well, it is interesting, but not so surprising." Feigenbaum: "But I found that bifurcations accumulate with the same exponential rate for different families." Moser: "What? Metrical invariant? This is impossible." The idea was still alien for mathematicians outside of a narrow group of mathematical physicists familiar with RG ideas. This story reminds us of the history of the Belousov-Zhabotinsky reaction. Belousov made his discovery of oscillatory regimes in chemistry in 1951. It took him almost 10 years to publish his results. The typical referee report would say: it is well known that time-periodic oscillatory regimes in chemistry are impossible. Finally, the paper appeared in 1959 in a nonrefereed conference proceedings. 
The discovery was fascinating and soon became very popular. Journalists were writing about universality in chaos, onset of chaos, universal route to chaos, etc. Experimentalists and numericists were finding more and more examples of physical systems exhibiting universality predicted by Feigenbaum's theory. While the theory is essentially one-dimensional, it was observed in many dissipative systems described by the finite-dimensional differential equations, and even infinite-dimensional PDEs. On the mathematical side $\mathrm{O}$. Lanford started his program on rigorous computer-assisted proofs of the existence of hyperbolic fixed points for renormalization. P. Collet, J.-P. Eckmann, and H. Koch extended the theory to the multidimensional dissipative case. In a joint paper with E. Vul, we constructed the thermodynamic formalism describing universal metrical properties of the Feigenbaum attractor. One should also mention the papers by M. Feigenbaum, L. Kadanoff, and S. Shenker and by D. Rand, S. Ostlund, J. Sethna, and E. Siggia where renormalization was studied in the context of critical circle maps. R. MacKay in his thesis applied a renormalization approach to invariant curves for area-preserving twist maps of the cylinder. The case of smooth invariant curves corresponds to the KAM regime. The most interesting was the case of critical invariant curves where MacKay numerically constructed a highly nontrivial fixed point. Rigorous computer-assisted results on the existence and the hyperbolicity of MacKay's fixed point were only obtained much later by $\mathrm{H}$. Koch. It is interesting to say a few words about the connection between the KAM theory and the renormalization. The first global result on the linearization of smooth circle diffeomorphisms with a typical irrational rotation number was proved by M. Herman around the time when Feigenbaum discovered the universality of renormalization. In fact, the linearization result can be viewed as a statement of convergence to the trivial renormalization fixed point given by linear maps with slope 1 . Here one can point at a parallel between the trivial linear fixed point of renormalization in dynamics, and trivial Gaussian fixed points in statistical mechanics. In fact, the linear fixed point is trivial, but a global convergence to it is a highly nontrivial result of Herman's theory. It is worth mentioning that local convergence was proved much earlier by V. Arnold (KAM-type result). We discuss local vs global convergence of renormalization below.

The Feigenbaum theory is based on a construction of a fixed point of the period-doubling transformation, and the proof that the linear operator corresponding to the linearization of this nonlinear transformation at the critical point has only one essential unstable eigenvalue-the famous Feigenbaum constant $4.6692 \ldots$, and the rest of the spectrum lies inside the open unit disk. The construction of the hyperbolic fixed point proves local universality of the period-doubling phenomenon. At the same time, it was clear from numerical experiments that the universality is, in fact, a much more general and more global phenomenon. Mathematical results in this direction only came much later, in the 1990s. The beautiful and highly nontrivial theory was developed in the papers by D. Sullivan, C. McMullen, and M. Lyubich. In fact, their results deal with a much more general setting than the case considered by Feigenbaum. A point of accumulation of perioddoubling bifurcations is the simplest instance of infinitely renormalizable maps. Roughly, it is a situation where one can precisely describe the combinatorial properties of the trajectory of the critical point. The map is supposed to be unimodal, that is, with a unique critical point (maximum) of order $\alpha$ ( $\alpha=2$ in the Feigenbaum case). The combinatorial information specifies, in particular, the sequence of times $t_{n}$ when the trajectory returns closest to the critical point. In the Feigenbaum case $t_{n}=2^{n}$. But one can consider a more general situation when $t_{n}=\prod_{i=1}^{n} k_{i}$, where the sequence $\left\{k_{i}, 1 \leq k_{i}<\infty\right\}$ characterizes different combinatorial types. The sequence of renormalization $R_{n}(f)$ can be naturally defined for any infinitely renormalizable unimodal map $f$. One of the main results of the renormalization theory for unimodal maps can be formulated the following way. Assume that $f$ and $g$ are two analytic infinitely renormalizable unimodal maps of the same combinatorial type, and with critical points of the same order $\alpha=2 l, l \in \mathbb{N}$. Then, the sequence of renormalization converges exponentially, namely $\left\|R_{n}(f)-R_{n}(g)\right\| \rightarrow 0$ as $n \rightarrow \infty$ exponentially fast.

The analyticity requirement can be relaxed. But basically all existing rigorous results assume that the order of a critical point is given by an even integer. At the same time it is largely believed and confirmed by numerical studies that the above result on exponential convergence of renormalization should remain true for any order $\alpha>1$. Proving such a result is probably the most important open problem in a general area around the Feigenbaum universality. The nature of the problem is similar for all $\alpha>1$ and can be described in the following way. Consider a part of the trajectory of the critical point in a small neighborhood of the critical point. The order of points in the past and in the future is determined by the combinatorial type. The metrical properties are defined by ratios of lengths of small intervals formed by the trajectory. The dynamics acting on these ratios/scalings consists of one instance of applying the map $|x|^{\alpha}$ and then many iterates far away from the critical point. The first step results in $O(1)$ changes of the scalings, and other iterates distort them only slightly. From this, one can determine new scalings corresponding to extending the trajectory to the future times. The 
description above is certainly too simplistic since in reality one has to take into account the distortions from a sequence of times when a trajectory comes back relatively close to the critical point. However one can see traces of universality here. Namely, the process we describe above does not use any information about the initial map apart from its combinatorial type and the order of the critical point. The main problem is to show that for a given infinitely renormalizable combinatorial type there exists only one set of scalings compatible with a given order of the critical point. In other words, the metrical properties of trajectories are extremely rigid.

The ideas of renormalization and universality pioneered by Mitchell Feigenbaum were hugely influential in dynamics. These ideas have really transformed and revolutionized the way we think about many dynamical problems. It is fair to say that renormalization became one of the most powerful methods of analyzing the asymptotic properties of dynamical systems, especially in the lowdimensional case. The convergence of renormalization is still a highly nontrivial problem. However, the big difference between renormalization in the dynamical systems setting and in statistical mechanics is that in many cases the dynamical renormalization theory can be developed rigorously. More examples of such rigorous development are discussed by M. Lyubich.

Back in the USSR. We turn now to more personal memories. It was probably in 1982 when Mitchell came to visit Moscow for the first time. The world was very different then. The Soviet Union looked super-stable, no one would have predicted its total collapse in less than 10 years. The visit was organized by the Landau Institute which was at its prime. It was an unbelievable group of physicists and mathematicians with very broad interests covering the whole of theoretical and mathematical physics. The atmosphere was extremely inspiring and creative. There was no pressure other than to do good research. Plus no grant applications, no committee meetings. Lots of intellectual freedom-it was really a paradise for young intellectuals. And young we were. A memorable talk of Sasha Migdal in 2004 at the meeting dedicated to Mitchell's 60th birthday at Rockefeller University gave a good description of the atmosphere at the Landau Institute at that time. The title of the talk-"Paradise Lost" - speaks for itself. Needless to say, Mitchell with his finely tuned soul felt very much at home in such an atmosphere. Many friendships which started then lasted for many years: L. Bunimovich, A. Migdal, A. Polyakov, to name a few. Endless discussions with topics ranging from literature, to history, to music. And science, of course. It was rewarding for everybody, including Mitchell, of course.
His next visit was in 1984 during the traditional LandauNordita meetings. This time Mitchell was part of the Nordita team which also included his close friend Predrag Cvitanović. It was a wonderful meeting with great personal interaction. Lots of drinks, lots of laughter, lots of deep late-night conversations. As Predrag wrote in his notes about the visit, Mitchell's diet was centered around caviar and champagne. This was for breakfast, most likely vodka was added in the evening. Mitchell had a very sharp eye. Once he noticed that in the local grocery store all the food was wrapped in paper rather than plastic. He immediately came to a conclusion that either all plastic is going to a military use, or there is no plastic at all. Several years later he was remembering this observation at the first sign of the unavoidable collapse of the Soviet Empire. Of course it was much before the modern times of "paper or plastic."

Then it was the next visit, perhaps in 1985, when one of us (KK) joined Mitchell on a very memorable three-day visit to Leningrad. Visits to the Hermitage Museum are always special, but going together with Mitchell made it even more unique. We went to LOMI (Leningrad's Branch of the Mathematical Institute) where Mitchell gave a talk. L. Faddeev, who was the director of LOMI, showed with great pride a beautiful grand piano which belonged to the Institute. Of course, Mitchell and Ludvig Faddeev both were great music lovers, and had much to talk about.

Next time we met in New York in December 1988. It was at a wonderful conference "Frontiers in Mathematics" organized by Joel Lebowitz and Peter Lax at the New York Academy of Sciences. Then it was a remarkable series of five(!) Soviet-USA conferences on chaos. The first (1989), the third (1991), and the fifth (1993) were organized in Woods Hole by David Campbell, Ken Ford, and Mitchell. The second (1990) and the forth (1992) were in the Soviet Union in Tarusa and Kiev. Well, by the time of the meeting, Kiev was already the capital of independent Ukraine. One of us (KK) was not able to participate in the last meeting at Woods Hall in 1993. Instead, a greeting message was sent to the participants which said that the series of meetings would be discontinued since it had achieved, in fact overachieved, its main purpose and goal: creating full and complete chaos in the Soviet Union. It took almost 25 years longer to achieve the same in the USA. The first of this series of meetings was really an amazing gathering of almost all leading experts in nonlinear dynamics and chaos theory, both mathematicians and physicists. The journal Chaos was the offspring of this meeting. Remarkably David Campbell, who founded it, served as the Editor-in-Chief of Chaos for 25 years.

Mitchell's wife Gunilla was an important part of his life. Almost immediately and very naturally she became a close friend. Gunilla was a talented artist, and Mitchell 
always proudly showed her latest paintings. There were many more get-togethers, mostly in their Manhattan apartment, sometimes in nearby restaurants. We remember many long conversations on different topics, as well as sometimes fierce arguments. Mitchell was an opinionated person, but you always had a feeling that he heard what you had to say.

Watching David Campbell and Mitchell together was great fun. They were very close, almost brothers, often arguing, always deeply feeling for each other. For us, Mitchell and David represented the best qualities of American intelligentsia. Although our life paths were very different, we had so much in common, so many things which attracted us to each other. It is always important to remember how universal the values which bring us together are.

Mitchell was a singular point. He lived a life of a thinker. He certainly needed moments of being alone. But he also loved life and loved his friends. He was deep and broad at the same time. A Renaissance man, one can say. Sadly, there are fewer and fewer people with such qualities. Are they becoming extinct? Mitchell will be always remembered and missed dearly.

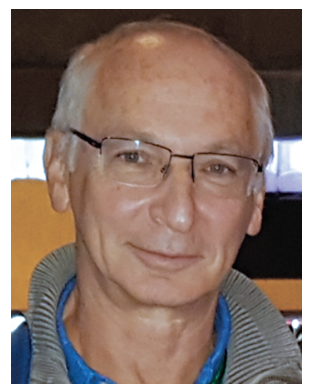

Konstantin Khanin

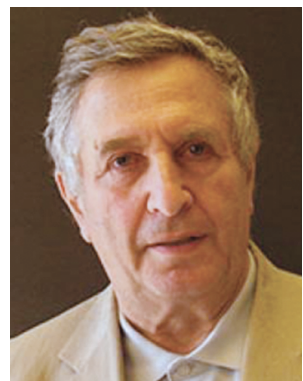

Yakov Sinai

\section{Renormalization Ideas in Dynamics: How Feigenbaum's Discovery Changed the World}

\section{Mikhail Lyubich}

Feigenbaum's discovery. In the mid 1970s, Feigenbaum accidentally made a curious observation. Using a hand calculator, he entertained himself by iterating simplest possible nonlinear maps: quadratic polynomials

$$
f_{a}: x \mapsto \operatorname{ax}(1-x)
$$

Mikhail Lyubich is a professor of mathematics at Stony Brook University and the director of the Institute for Math Sciences at Stony Brook. His email address is m7yubich@math. stonybrook. edu.

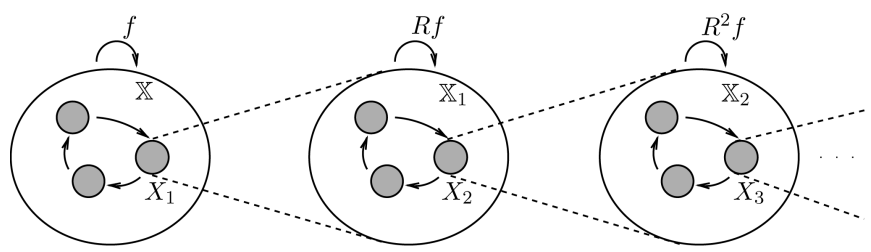

Figure 1. Renormalization telescope.

on the interval $[0,1]$. He observed that for small $a>0$, almost all orbits converge to an attracting fixed point. Then at some parameter $a_{1}$, the doubling bifurcation happens, making almost all orbits converge to an attracting cycle of period 2. This persists for some interval of parameters, until the next doubling bifurcation occurs, making almost all orbits converge to an attracting cycle of period 4 , etc. This cascade of doubling bifurcation parameters $\left(a_{n}\right)$ converges to a certain parameter $a_{F}$, called now the Feigenbaum point. Feigenbaum noticed that the convergence happens rapidly, namely exponentially fast with rate $\lambda=4.6 \ldots$. He took notice of this number.

Sometime later he played the same game with a similar family $g_{b}: x \mapsto b \sin x$ and observed a similar cascade of bifurcations $\left(b_{n}\right)$ converging exponentially fast to some parameter $b_{F}$. He looked at the rate and was struck to discover that it was the same: $\lambda=4.6 \ldots$. He immediately realized that there should be a deep reason behind this seeming coincidence (see $[\mathrm{F}])$.

Ideas coming from physics (quantum field theory and statistical mechanics) helped him to reveal the underlying mechanism for this universality phenomenon. ${ }^{1}$ It is called renormalization, a machinery relating various scales of a family of dynamical systems. It has become a powerful tool of the dynamical system theory, radically changing the course of its further development, particularly in the low-dimensional world.

General idea of renormalization. We will give a very general idea of renormalization in dynamics. Let us consider a dynamical system $f: \mathbb{X} \rightarrow \mathbb{X}$ of a certain class $Q$. To understand its small-scale structure, let us select a piece $X_{1} \subset \mathbb{X}$ and consider the first return map $f_{1}: X_{1} \rightarrow X_{1}$ If we are lucky and careful, this return map may belong to the original class $Q$, so we may try to compare the new map $f_{1}$ to the original map $f$. To this end, we rescale the small piece $X_{1}$ to the "original size" by some change of variable $h_{1}: X_{1} \rightarrow \mathbb{X}_{1}$ (which may or may not be linear). Conjugating $f_{1}$ by $h_{1}$, we obtain a new map $R f: \mathbb{X}_{1} \rightarrow \mathbb{X}_{1}$ called the renormalization of $f$. This map describes the dynamics of $f$ in scale $X_{1}$.

\footnotetext{
${ }^{1}$ About at the same time, Coullet and Tresser [TC] realized that there is a similar Universality phenomenon in the dynamical space: e.g., the postcritical sets of the Feigenbaum maps $f_{a_{F}}$ and $g_{b_{F}}$ have the same Hausdorff dimension.
} 
It may happen that the map $R f$ is again renormalizable in the same sense, yielding the second renormalization $R^{2} f$ : $\mathbb{X}_{2} \rightarrow \mathbb{X}_{2}$, which is the rescaled return map $f_{2}: X_{2} \rightarrow X_{2}$ in a smaller scale $X_{2} \subset X_{1}$. It describes the dynamics of $f$ in that scale.

With some luck, we can continue this process many times yielding a sequence of renormalizations $R^{n} f$ in a nest of scales. It may even happen that this sequence is infinite, giving us a description of the dynamics in all scales. In this case, the original map is called infinitely renormalizable.

However, this small-scale description is topological at best, unless we have some uniform geometric control of all the renormalizations. Such control, called a priori bounds, amounts to precompactness of the family of renormalizations $R^{n} f$ in $Q$. To prove such a priori bounds is usually a difficult technical problem. But it is rewarding as we gain uniform geometric control of the original system in all scales.

But the renormalization program is even more ambitious. It suggests that under some favorable circumstances there may exist a renormalization fixed point $f_{*} \in Q, R f_{*}=f_{*}$. Such a map is homogeneous in all scales, and hence selfsimilar. This conjecture does not come from anywhere except physical intuition originated in the statistical mechanics and quantum field theory. This explains why physicists (Feigenbaum, Coullet, Tresser, Cvitanović, Kadanoff,...) were a driving force in the initial stages of the renormalization theory.

In fact, physical intuition, accompanied with computer experiments, suggested even more: that this fixed point $f_{*}$ is hyperbolic under the renormalization, in the usual dynamical sense. This means that there exist two transverse manifolds, stable manifold $\mathcal{W}^{s}$ and unstable manifold $\mathcal{W}^{u}$, with the following properties:

- $\mathcal{W}^{s}$ is forward invariant under $R$ and the orbits $R^{n} f$ in $\mathcal{W}^{s}$ converge to $f_{*}$ exponentially fast. (This manifold consists of infinitely renormalizable maps.)

- $\mathcal{W}^{u}$ is backward invariant under $R$ and the orbits $R^{-n}$ in $\mathcal{W}^{u}$ converge to $f_{*}$ exponentially fast. Moreover, the unstable manifold is finite-dimensional, with dimension equal to the number of "essential" parameters in our space $Q$.

Thus, the small-scale structure of any infinitely renormalizable map $f \in \mathcal{W}^{s}$ coincides (up to an exponentially small error) with the fixed point $f_{*}$. In particular, all these maps are asymptotically self-similar. This phenomenon is called the dynamical universal self-similarity of the maps of our class.

On the other hand, the unstable manifold $\mathcal{W}^{u}$ represents a nontrivial finite-parameter family of maps invariant under $R^{-1}$. If it is one-dimensional, then the bifurcation locus in this family contains a rescaled copy of itself (in an appropriate coordinate), representing parameter selfsimilarity.

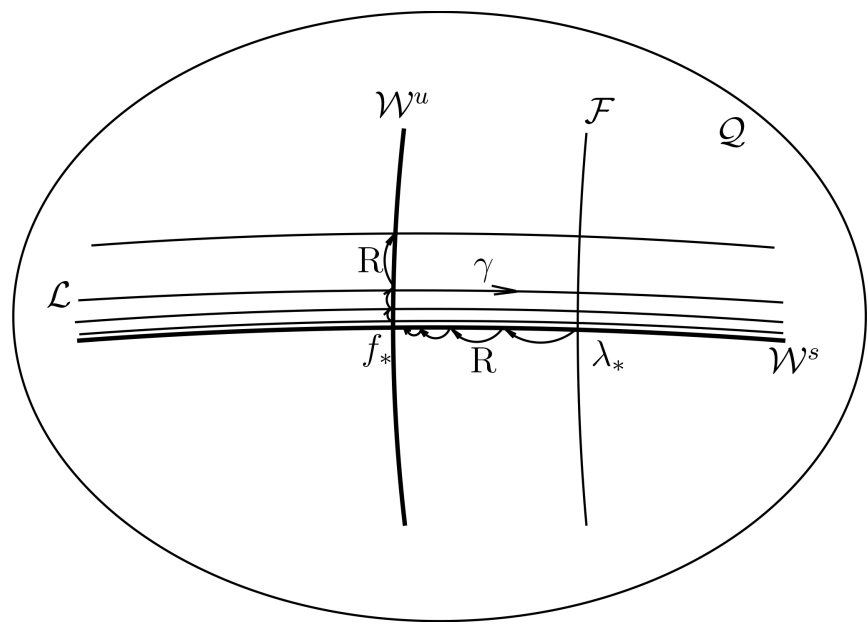

Figure 2. Renormalization picture behind self-similarity, rigidity, and universality.

In the presence of some extra structure, the parameter self-similarity also becomes universal. Namely, imagine that certain topologically defined classes form a codimension-one lamination $\mathcal{L}$ in the space $\mathcal{Q}$ invariant under the renormalization. Then any one-parameter family $\mathcal{F}=\left(f_{\lambda}\right)$ of maps in $\mathcal{Q}$ which transversely intersects $\mathcal{W}^{s}$ (at some parameter $\lambda_{*}$ ) will be related to $\mathcal{W}^{u}$ via the holonomy $\gamma$ along $\mathcal{L}$. This makes the bifurcation loci in $\mathcal{F}$ and $\mathcal{W}^{u}$ homeomorphic.

Moreover, for general dynamical reasons, $\gamma$ is asymptotically conformal at $\lambda_{*}$, making those bifurcation loci (asymptotically) conformally equivalent near the corresponding points, $\lambda_{*}$ and $f_{*}$. This is the parameter universality we have alluded to above.

In practical terms, if you observe self-similarity of the bifurcation picture of some family of dynamical systems (e.g., the quadratic family), then quite likely it is generated by some renormalization mechanism. Feigenbaum's original observation gave the first glimpse into this fundamental phenomenon.

Mathematics that came out of it.

Doubling renormalization. The specific scheme responsible for the Feigenbaum discovery is the period doubling renormalization in the space of unimodal maps with quadratic criticality (i.e., interval maps with a single nondegenerate critical point, which we place at 0 ). If such a map has a periodic interval $I^{1} \ni 0$ of period two, then $f^{2} \mid I^{1}$ is also a unimodal map. Rescaling $I^{1}$ back to the unit size defines a renormalization transformation $R$ in the space $\mathcal{U}$ of unimodal maps. If a map is infinitely renormalizable, then it possesses a nest of intervals $I^{1} \supset I^{2} \supset I^{3} \supset \cdots$ of periods $2^{n}$, representing various renormalization scales. In this situation, the renormalization conjecture suggests that there exists an infinitely renormalizable map $f_{*}$ such that $f_{*}^{2^{n}} \mid I^{n}$ 
are obtained one from another via scaling by a certain factor $\rho=-2.7 \ldots$. Moreover, for any infinitely renormalizable map $f$, the scaling of the corresponding renormalization intervals will asymptotically be the same, and such maps form a codimension-one submanifold $\mathcal{W}^{s}$ in $\mathcal{U}$. In the transverse direction to $\mathcal{W}^{s}$, there is a one-dimension unstable manifold $\mathcal{W}^{u}$ on which $R$ acts (in an appropriate coordinate) as a scaling by some factor $\lambda=4.6 \ldots$. The same scaling rate is observed in any one-parameter family of unimodal maps transverse to $\mathcal{W}^{s}$, e.g., in the quadratic family. It is the universal rate originally observed by Feigenbaum.

Shortly afterwards, similar universality phenomena were observed for more general renormalization schemes in the space of unimodal maps, with various periods. Then experiments, physical and numerical, indicated that this phenomenon (sometimes with the same scaling factors as in the unimodal setting) is also observed in higher-dimensional situations, including real fluid motions. Gradually the universality was establishing itself as a new world paradigm.

Proof of the renormalization conjecture. From the physical point of view, the unimodal story looked quite complete: there was a coherent theory clearly explaining experimental observations. However, the mathematical mechanism behind this theory and its rigorous justification did not look so obvious. In 1982, Lanford gave the first rigorous proof of the doubling renormalization conjecture, relying heavily on rigorous computational estimates. Various pieces of the theory were then supplied, without appealing to computers, by H. Epstein, J.-P. Eckmann, K. Khanin, Ya. Sinai, and other people (see [VSK]).

At about the same time (in the early 1980s), similar phenomena were observed in the holomorphic world. It manifested itself by self-similar features of the Mandelbrot set $\mathcal{M}$ where one could observe little copies that were indistinguishable from $\mathcal{M}$ itself (see Figure 3). A mathematical justification for the existence of these copies was given on a topological level by Douady and Hubbard in the mid-1980s, and it was based upon an idea of quadraticlike renormalization. It is a holomorphic counterpart of the unimodal notion, where the underlying space $\mathcal{Q}$ consists of holomorphic double branched coverings $f: U \rightarrow V$ from a smaller topological disk to a bigger one.

In his address to the Berkeley ICM (1986) Dennis Sullivan articulated that the holomorphic Douady-Hubbard quadratic-like setting provides a natural mathematical frame for the renormalization theory, supplying it with a wealth of geometric structures and technical tools unavailable in the real world. He specifically outlined an approach to the construction of the renormalization fixed point $f_{*}$ and its stable manifold $\mathcal{W}^{s}$ based upon Teichmüller theory ideas (see [S, MvS]). Then Curt McMullen

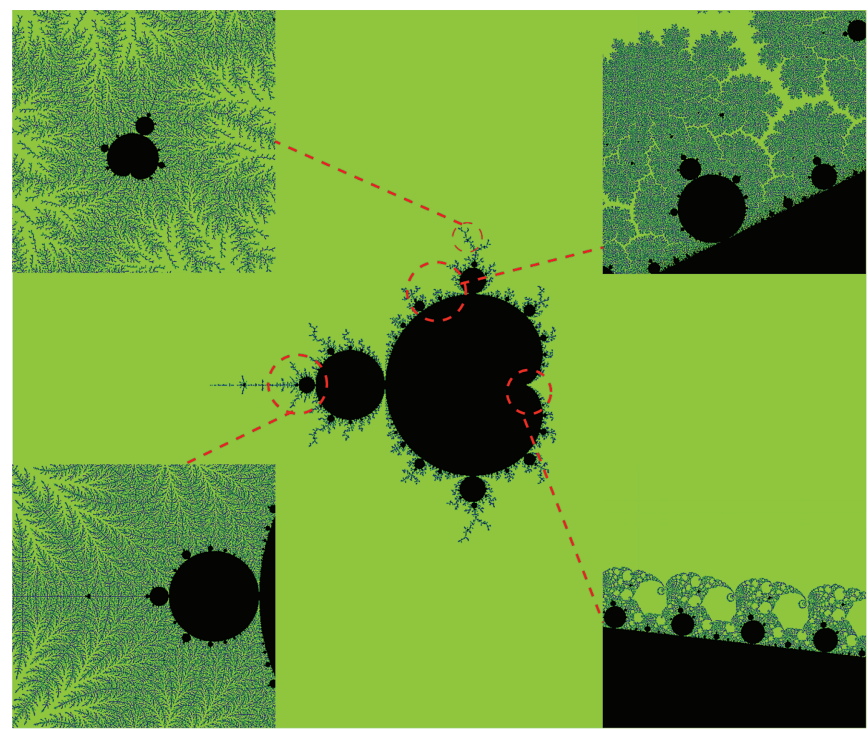

Figure 3. Self-similarity of the Mandelbrot set in several places. The lower-left corner corresponds to the classical Feigenbaum parameter that initiated the whole story. The upper-right corner corresponds to its close relative, the golden mean Siegel parameter.

had an insight into the problem from the point of view of hyperbolic 3D geometry, which allowed him to justify that the renormalization orbits in the stable manifold converge to the fixed point exponentially fast [McM]. The story was completed in [L1] with a proof of the existence of the $1 \mathrm{D}$ unstable manifold $\mathcal{W}^{u}$ transverse to $\mathcal{W}^{s}$ which justified the parameter universality for any unimodal combinatorics. Several years later, the Sullivan-McMullen part of the story was revisited by Artur Avila and the author with ideas of Carathéodory metric and almost periodicity [AL]. Regular or stochastic theorem. The author went on to prove the renormalization conjecture for all combinatorial types simultaneously [L2]. This was a crucial piece of the Regular or Stochastic Theorem asserting that for almost any $c \in[-2,1 / 4]$, the quadratic polynomial is either regular (i.e., almost all orbits on the invariant real interval $\mathcal{J}_{c}$ converge to an attracting cycle) or stochastic (i.e., the behavior of almost all orbits in $\mathcal{J}_{c}$ is governed by an absolutely continuous invariant measure). This gives a complete dynamical picture in the real quadratic family from the probabilistic viewpoint, and renormalization provided key insights into the matter.

Impact on 1D holomorphic dynamics. A central problem of holomorphic dynamics is the MLC conjecture asserting that the Mandelbrot set $\mathcal{M}$ is locally connected. If true, it would supply us with a precise topological model for this tremendously complex fractal set and would resolve another outstanding problem, the Fatou conjecture 
asserting that hyperbolic maps ${ }^{2} f_{c}: z \mapsto z^{2}+c$ are dense in the Mandelbrot set.

Around 1990, Yoccoz made a breakthrough in the MLC (related to an earlier work by B. Branner and J.-H. Hubbard) by proving that $\mathcal{M}$ is locally connected at $c$ unless $f_{c}$ is infinitely renormalizable. This result tightly linked the MLC conjecture to renormalization theory. Further advances in the MLC problem fit perfectly into the renormalization worldview (see work by Lyubich, Shishikura, Kahn, Cheraghi, and Dudko over the past 25 years).

But the impact of the renormalization ideas on holomorphic dynamics goes beyond the Douady-Hubbard framework. Indeed several other renormalization themes have been designed that give insight into self-similarity of various parts of the Mandelbrot set (see Figure 3). Siegel-Pacman renormalization theory, also originating in physics, is responsible for the self-similarity near the golden mean point on the main cardioid (McMullen, Yampolsky, Dudko-Lyubich-Selinger; see [DLS] and references therein), while the parabolic renormalization theory controls the geometry of $\mathcal{M}$ near the cusp and other parabolic points (Lavaurs, Douady, Inou-Shishikura; see [IS] and references therein).

Without a doubt, all these theories will play an important role in the MLC story. And remarkably, they have already provided a key to other outstanding problems. For instance, they were crucial for constructing examples of Julia sets of positive area (Buff-Cheritat, Avila-Lyubich).

It is hard to overestimate the influence that the renormalization ideas had on the development of the onedimensional dynamics, real and complex.

Other themes. To indicate the scope of the theory, let us mention in conclusion several other themes greatly influenced by renormalization ideas:

- Circle diffeomorphisms. This theme goes back to Arnold's classical work, followed up by M. Herman, J.-C. Yoccoz, K. Khanin \& Ya. Sinai, and many others. A general renormalization picture was recently described by N. Goncharuk and M. Yampolsky [GY]. It also branched off to the theory of piecewise circle diffeomorphisms with break points (Khanin, Kocic, and others).

- Critical circle maps. Also initiated by physicists (Feigenbaum, Kadanoff, Siggia,...), this renormalization picture turns out to be closer to the unimodal renormalization than to renormalization of circle diffeomorphisms. It has been developed by G. Swiatek, M. Herman, E. De Faria, W. de Melo, M. Yampolsky, and others. Now the theory is complete; see $[\mathrm{Y}]$ and references therein.

- Interval exchange maps and Teichmüller flow. Another direction branched off the renormalization theory of circle diffeomorphisms is the Rauzy renormalization for interval exchange maps and its continuous counterpart, the Teichmüller flow acting on the space of flat surfaces. It has been flourishing in the past two decades, with many remarkable results by $\mathrm{H}$. Masur, W. Veech, A. Zorich, M. Kontsevich, G. Forni, C. McMullen, A. Avila, M. Viana, M. Mirzakhani, A. Eskin, and many others; see [EMM].

- Schrödinger cocycles. This theory arose directly from quantum mechanical spectral theory for the Schrödinger operator with almost periodic potential. Mathematically, it amounts to the study of $\operatorname{SL}(2, \mathbb{R})$-cocycles over circle rotations. It has also experienced an explosive development over the past two decades, with deep contributions by A. Avila, R. Krikorian, S. Zhitomirskaya, J. Bourgain, M. Goldstein, W. Schlag, and many others. A complete global bifurcation picture, in the spirit of the aforementioned Regular or Stochastic Theorem, was described by A. Avila [Av]. Renormalization of cocycles fibered over the circle renormalization played a crucial role in this development.

- Dissipative Hénon maps. This renormalization theory has been developed in the work of H. Koch, S. van Strien, C. Tresser, A. de Carvalho, M. Lyubich, M. Martens, and other people as a two-dimensional perturbation of the unimodal renormalization theory. In this world the rigidity and universality phenomena appear in a much more subtle form than in the one-dimensional context; see [CLM].

- Conservative dynamics. This theory includes conservative Hénon maps as well as Hamiltonian systems (with the KAM theory fitting together well with the renormalization framework). Though the evidence for universality is plentiful, it is much more difficult to justify it rigorously, and usually it needs substantial computer assistance. The work by R. McKay, H. Koch, D. Gaidashev, among others, gives a sample of this important area of research; see [K].

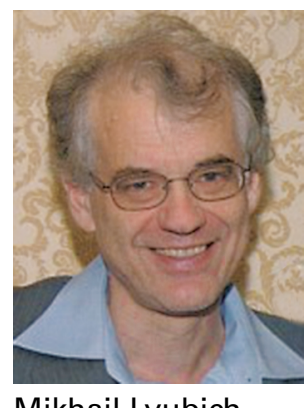

Mikhail Lyubich

\footnotetext{
${ }^{2}$ In this context, they can be defined as maps possessing an attracting cycle.
} 


\section{Feigenbaum's Years at Cornell and Rockefeller}

\section{Eric D. Siggia}

There was a fruitful convergence of mathematics and physics in the late 1970s and 1980s on the question of how fluids pass from laminar to turbulent flow and in particular if the transition is discrete. Mitchell's singular contribution anticipated this convergence but was not the only antecedent. A decade prior, the physicists who were drawn to this question, from both experiment and theory, studied thermodynamic phase transitions, and the exacting experimental techniques required to uncover the scaling behavior around second-order critical points proved very useful in the new study of nonequilibrium transitions ([Ah], https://digitalcommons.rockefeller . edu/1ibchaber-1aboratory/1/). On the theory side, it's fair to say that the subject of critical point scaling was "solved" by Ken Wilson's Renormalization Group 19691971 , and a certain number of us were looking for other problems to solve.

A snapshot of the period was the program at the Physics Institute at the University of California at Santa Barbara in 1980-81. Mathematical physicists such as Ruelle and Eckmann were attending, but there was also a strong contingent from experimental physics. Equal time was given to spatially extended systems that have not attracted any mathematical attention. I mention this program since it was my first extended exposure to Mitchell, and suffice it to say that he was not happy in the beach culture of Santa Barbara. Our professional lives converged during this period. With British mathematician David Rand, and two younger colleagues, we worked out the onset of chaos in circle maps with arbitrary winding number by renormalization group methods [ÖRSS]. Completely independently, Leo Kadanoff in Chicago and a student Scott Shenker (now UC Berkeley) with assistance from Mitchell did similar work [FKS]. Shenker was later a postdoc with Mitchell and me at Cornell and remains in awe of Mitchell's aesthetics and science.

The year after the Santa Barbara meeting, I invited Mitchell to Cornell, to give a series of lectures on iterated maps and period doubling. The Cornell physics department has a bipartite structure of two labs: one particle physics where Mitchell was a postdoc a decade prior,

Eric D. Siggia is the Viola Ward Brinning and Elbert Calhoun Brinning Professor at Rockefeller University. His email address is siggiae@mai1 . rockefe11er.edu. and the other condensed matter. Ken Wilson and Michael Fisher bridged this institutional divide with famous results in the 1970s and Mitchell was hired with super-laboratory status, though his office was in condensed matter. There he quickly noted the significance of my Cornell extension: 255-4669. Ithaca more than now was a village, and I can recall lunches with Ken Wilson and numerous dinners at a local Italian restaurant where rabbit was the specialty. Relations with the campus community who were studying turbulence in engineering contexts were correct but frosty.

Mitchell's schedule did not accommodate morning classes, nor did his work habits tolerate the divided attentions required of university life. His obsession with working every subject from the ground up consumed a lot of time, but it was more than many students could appreciate. So by 1987, he was the Toyota Professor at Rockefeller University in NYC where he moved with his extraordinary Swedish born wife Gunilla who matched his aesthetics. She had multiple artistic talents, but her paintings that evolved through distinct phases were the most enduring image I retain from their apartment at $450 \mathrm{E}$ 63rd street.

Rockefeller University, founded in 1901, was a biomedical research institute until 1954 when it became a university with only graduate students and later broadened its mandate to include physics, mostly theoretical, and philosophy. There was no mandatory teaching, no defined duties, and no need for grant support if the group was small. So for Mitchell, this was nirvana. He was hired by the then president Joshua Lederberg, who had the strongest claim to polymath status among the founders of molecular genetics, and Mitchell witnessed the turbulent administration of David Baltimore. During this period, the financial crisis of the 80 s caused a retrenchment, and it was decided to focus on biomedical research. Faculty in other areas drifted away; the particle theorist A. Pais stayed and became a noted historian of modern physics.

Rockefeller remained, however, a singular institution. Only there could Mitchell Feigenbaum forge a distinguished career as an administrator. He did so with the aid of the next president, Torsten Wiesel, a neurobiologist who uncovered the early steps of visual processing in mammals. Torsten tried to interest Mitchell in animal experiments, but wrote that Mitchell's world was elsewhere. Faced with the institutional disappearance of physics, Mitchell sold to Wiesel and the trustees the then very novel idea of seeding the university with a few physical scientists with nascent interests in biology but above all a strong professional grounding. The people he recruited, Albert Libchaber in 1996, me in 1997, and finally a junior hire, Marcelo Magnasco, had all passed through nonlinear dynamics. (Libchaber did the precision experiments confirming period doubling in convection.) In retrospect, our common bond 
was not that we were a clan, but rather those who left conventional physics for nonlinear dynamics were more apt to move again to biology. It's fair to say none of us knew much about biology. The hope for the "Center of Studies in Physics and Biology" as expressed by Wiesel was to provide Rockefeller with a toehold in sciences outside of biology, particularly theory. It has become commonplace to welcome quantitative methods in biology settings, but too often the implicit motivation is high-level tech support, not the intellectual direction, as envisioned by Mitchell and Torsten.

Mitchell seized the right moment at Rockefeller to implant physics (though his hope to include serious mathematics was never realized). His access to the administration died when Wiesel was replaced by Levine (who added another physicist, Stan Leiber, with better biological credentials than the three first hires). Perhaps the strongest institutional imprint of the Rockefeller center has been the fellows program. These are three-year appointments with no implicit attachment to any faculty and a mandate to find an interesting project in the Rockefeller context. Mitchell always participated in fellow interviews until he became ill, though decisions were made by vote of the center faculty and current fellows.

It's fair to say that institutional interest in the physics center was moribund by the turn of the millennium. As Libchaber aptly expressed it, we were the "Jews of Rockefeller." But life was good in the ghetto. At Mitchell's initiative, residents of the center besides the fellows and itinerant visitors to New York grew to include a noted historian of psychiatry from Cornell, and a cultural anthropologist from Denmark with UN connections. We enjoyed ourselves, gradually becoming more biological and forging collaborations with a subset of labs willing to entertain our divergent and disruptive questions.

Mitchell had his 60th birthday in 2004 and celebrated with typical aplomb by a two-day symposium at Rockefeller entitled "Numbers and Nature." The cast of characters was wide: Michael Berry, Leo Kadanoff, Sasha Polyakov, and others from physics and astronomy, David Ruelle, Yakov Sinai, and Dennis Sullivan from dynamical systems and beyond, plus a leavening of humanists with whom Michell had engaged in the past. The group cohered at this event; Mitchell was clearly the sun around which it revolved, but he in no way dominated the proceedings. Rather I feel he just observed how the pieces fell together. Mitchell could be arrogant and opinionated but was always generous, enjoyed an argument, and never held a grudge. His diverse circle of friends was a testimony to his character and charisma, and their devotion was manifest when he became ill.
Mitchell's final major project was a book on optics, more precisely, anamorphs and is aptly entitled Reflections on a Tube. These are planar images viewed reflected in a cylindrical mirror to recreate a recognized object. But what is perceived is not the result of geometric optics since the eye is not a pinhole camera. One has to compute the caustics and the subtle way they influence cognition with additional connections made to the ophthalmology of vision. A subject that appears arcane actually requires going to the base of our visual processing machinery. The project began in 2006, but the final editorial work necessary for publication was not completed by Mitchell, and the task has been assumed by a colleague who taught from the book.

Mitchell burned the candle of life from both ends with full acceptance of the possible consequences. Smoking enhanced focus so he smoked. In his final year after the diagnosis of throat cancer, I saw more of him than any time since our Cornell days. He was divorced from Gunilla by then but they remained in contact. His friends in New York and beyond saw him through the ordeal. He was ostensibly cured of the cancer, but died from the collateral damage of the treatment. It was an ordeal; Mitchell researched his condition in typical fashion and would not readily countenance mushy medical pronouncements. His voice deteriorated which pained him, he was despondent and sometimes defiant but never displayed the apathy of depression. He was in a situation from which he could not think his way out.

Wiesel wrote "Mitch Feigenbaum was a true genius and his presence as professor at the Rockefeller University will always be part of its proud heritage." An oral history interview from August 2018 is preserved in https://digitalcommons.rockefe11er.edu /feigenbaum-7aboratory/2/cd.

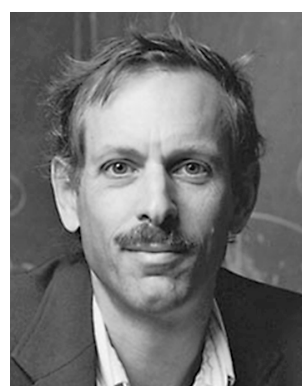

Eric D. Siggia 


\section{References}

[Ah] G. Ahlers, Over two decades of pattern formation, a personal perspective, in 25 Years of Non-Equilibrium Statistical Mechanics, vol. 445, no. 2, Springer, Berlin and Heidelberg, 1995, pp. 91-124.

[Av] Artur Avila, Global theory of one-frequency Schrödinger operators, Acta Math. 215 (2015), no. 1, 1-54, DOI 10.1007/s11511-015-0128-7. MR3413976

[AL] Artur Avila and Mikhail Lyubich, The full renormalization horseshoe for unimodal maps of higher degree: exponential contraction along hybrid classes, Publ. Math. Inst. Hautes Études Sci. 114 (2011), 171-223, DOI 10.1007/s10240-011-0034-2. MR2854860

[CLM] A. De Carvalho, M. Lyubich, and M. Martens, Renormalization in the Hénon family. I. Universality but nonrigidity, J. Stat. Phys. 121 (2005), no. 5-6, 611-669, DOI 10.1007/s10955-005-8668-4. MR2192529

[DLS] Dzmitry Dudko, Mikhail Lyubich, and Nikita Selinger, Pacman renormalization and self-similarity of the Mandelbrot set near Siegel parameters, J. Amer. Math. Soc. 33 (2020), no. 3, 653-733, DOI 10.1090/jams/942. MR4127901

[EMM] Alex Eskin, Maryam Mirzakhani, and Amir Mohammadi, Isolation, equidistribution, and orbit closures for the $\mathrm{SL}(2, \mathbb{R})$ action on moduli space, Ann. of Math. (2) 182 (2015), no. 2, 673-721, DOI 10.4007/annals.2015.182.2.7. MR3418528

[F] Mitchell J. Feigenbaum, Quantitative universality for a class of nonlinear transformations, J. Statist. Phys. 19 (1978), no. 1, 25-52, DOI 10.1007/BF01020332. MR501179

[FKS] Mitchell J. Feigenbaum, Leo P. Kadanoff, and Scott J. Shenker, Quasiperiodicity in dissipative systems: a renormalization group analysis, Phys. D 5 (1982), no. 2-3, 370-386, DOI 10.1016/0167-2789(82)90030-6. MR680571

[GY] N. Goncharuk and M. Yampolsky, Analytic linearization of conformal maps of the annulus, arXiv:2004.05126, 2020.

[IS] H. Inou and M. Shishikura, The renormalization for parabolic fixed points and their perturbations, manuscript, 2008, www.math. kyoto-u.ac.jp/ mitsu/pararenorm/.

[K] Hans Koch, Renormalization of vector fields, Holomorphic dynamics and renormalization, Fields Inst. Commun., vol. 53, Amer. Math. Soc., Providence, RI, 2008, pp. 269330. MR2477427

[L1] Mikhail Lyubich, Feigenbaum-Coullet-Tresser universality and Milnor's hairiness conjecture, Ann. of Math. (2) 149 (1999), no. 2, 319-420, DOI 10.2307/120968. MR1689333

[L2] Mikhail Lyubich, Almost every real quadratic map is either regular or stochastic, Ann. of Math. (2) 156 (2002), no. 1, 1-78, DOI 10.2307/3597183. MR1935840

[McM] Curtis T. McMullen, Renormalization and 3-manifolds which fiber over the circle, Annals of Mathematics Studies, vol. 142, Princeton University Press, Princeton, NJ, 1996. MR1401347
[MvS] Welington de Melo and Sebastian van Strien, Onedimensional dynamics, Ergebnisse der Mathematik und ihrer Grenzgebiete (3) [Results in Mathematics and Related Areas (3)], vol. 25, Springer-Verlag, Berlin, 1993. MR1239171

[ÖRSS] Stellan Östlund, David Rand, James Sethna, and Eric Siggia, Universal properties of the transition from quasiperiodicity to chaos in dissipative systems, Phys. D 8 (1983), no. 3, 303-342, DOI 10.1016/0167-2789(83)90229-4 MR719630

[S] Dennis Sullivan, Bounds, quadratic differentials, and renormalization conjectures, American Mathematical Society centennial publications, Vol. II (Providence, RI, 1988), Amer. Math. Soc., Providence, RI, 1992, pp. 417-466. MR1184622

[TC] Charles Tresser and Pierre Coullet, Itérations d'endomorphismes et groupe de renormalisation (French, with English summary), C. R. Acad. Sci. Paris Sér. A-B 287 (1978), no. 7, A577-A580. MR512110

[VSK] E. B. Vul, Ya. G. Sina1, and K. M. Khanin, Feigenbaum universality and thermodynamic formalism (Russian), Uspekhi Mat. Nauk 39 (1984), no. 3(237), 3-37. English transl., Russian Math. Surveys 39 (1984), 1-40. MR747790

[Y] Michael Yampolsky, Hyperbolicity of renormalization of critical circle maps, Publ. Math. Inst. Hautes Études Sci. 96 (2002), 1-41 (2003), DOI 10.1007/s10240-003-0007-1. MR1985030

Credits

Photo of Mitchell Feigenbaum is courtesy of Gemunu Gunaratne and Predrag Cvitanović.

Figures 1 and 2 are courtesy of Lilya Lyubich.

Figure 3 was made by Dzmitry Dudko using W. Jung's Mandel program.

Photo of Konstantin Khanin is courtesy of Konstantin Khanin.

Photo of Mikhail Lyubich is courtesy of Mikhail Lyubich.

Photo of Eric Siggia is courtesy of Eric Siggia.

Photo of Yakov Sinai is courtesy of Yakov Sinai. 